Fragmentierung der $\beta$ - bzw. $\gamma$-Bindung erwarten. Dabei sollte sowohl wegen des Masseneffektes als auch wegen der Ladungsstabilisierung am tertiären Kohlenstoffatom die positive Ladung bevorzugt beim $\mathrm{C}_{3} \mathrm{H}_{7}$-Radikal bleiben. Außerdem sollte die Ladungsverschiebung im elektrischen Feld ebenfalls dieses Fragmention gegenüber dem $\mathrm{C}_{4} \mathrm{H}_{9}{ }^{+}$-Ion begünstigen. Ferner sollten die Massen 15 und M-15 stärker als beim n-Heptan ausgeprägt sein. Im Experiment werden diese Erwartungen bestätigt (s. Abb. 1).

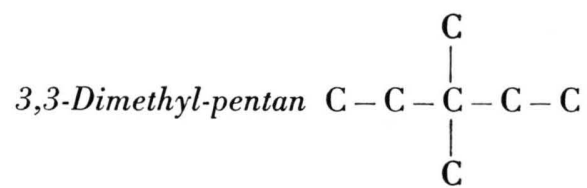

Für das 3,3-Dimethyl-pentan wird man wieder eine Fragmentierung der $\beta$-Bindung erwarten. Dabei können die Ionen der Masse 71 und 29 auftreten. Es ist jedoch nicht eindeutig vorherzusagen, an welchem der beiden Bruchstücke die Ladung besser stabilisiert sein wird. Bleibt sie am zentralen Kohlenstoffatom, so ist sie durch die Polarisation dreier Substituenten stabilisiert. Ein bevorzugtes Auftreten des $\mathrm{C}_{2} \mathrm{H}_{5}{ }^{+}$. Ions wäre durch eine Verschiebung der Ladung im Feld begünstigt, wenn man annimmt, daß das Molekül in Längsrichtung vor der Spitze ausgerichtet ist. Da es sich aber um ein relativ kugelförmiges Molekül handelt, dürfte keine sehr große Wahrscheinlichkeit für eine bestimmte Orientierung im Feld gegeben sein, da die Anisotropie der Polarisierbarkeit in den beiden Molekülachsen gering ist. Es wird beobachtet, daß das $\mathrm{C}_{2} \mathrm{H}_{5}{ }^{+}$-Ion erst bei hohen Feldstärken die Intensität des $\mathrm{C}_{5} \mathrm{H}_{11}{ }^{+}$-Ions erreicht und übertrifft. Ebenfalls werden relativ intensive Massenlinien bei $m / e=15$ und $m / e=85(M-15)$ gefunden. Die übrigen Massenlinien, die sonst ebenfalls bei n-Paraffinen erscheinen und hier mit einer geringen Intensität auftreten, sind auf isomere Verunreinigungen zurückzuführen.

Der Deutschen Forschungsgemeinschaft sowie dem Verband der Chemischen Industrie danken wir für die großzügige Förderung der vorliegenden Arbeit durch finanzielle Unterstützung.

\title{
Feldionen-Massenspektren organischer Moleküle IV. Olefine
}

\author{
H. D. Beckey und P. Schulze \\ Institut für Physikalische Chemie der Universität Bonn \\ (Z. Naturforschg. 20 a, 1335-1344 [1965] ; eingegangen am 21. Juli 1965)
}

\begin{abstract}
The field ion mass spectra of the olefins are discussed. It is shown that the double bond and the bond next to it are very stable against field dissociation. The fragment patterns of all olefins show some features due to the stable configuration of the allyl radical or the allyl ion respectively. This is verified by some energetic calculations, by which the spectra of some olefins are explained, too. The stabilities of paraffins and olefins against field dissociation are compared.
\end{abstract}

BECKEY und $\mathrm{W}_{\mathrm{AgNER}}{ }^{1}$ haben bereits angedeutet, daß 1-Olefine bei Feldionisierung zu stärkerer Fragmentierung neigen als n-Paraffine, auf der anderen Seite jedoch die $\mathrm{C}=\mathrm{C}$-Doppelbindung sich ähnlich stabil verhält wie die $\mathrm{C}-\mathrm{H}$-Bindung in den Paraffinen. WAGNER $^{2}$ hat darüber hinaus das Feldionen(FI) Massenspektrum des 2,4,4-Trimethyl-1-penten und des 2,4,4-Trimethyl-2-penten angegeben und bei einem Vergleich festgestellt, daß das zweite Molekül gegenüber Fragmentierung stabiler ist. Weitere und

\footnotetext{
1 H. D. Beckey u. G. Wagner, Z. Analyt. Chem. 197, 58 [1963].

2 G. WAGNer, Dissertation, Techn. Hochschule Aachen 1963.

${ }^{3}$ H. D. BECKEY, Z. Naturforschg. 17 a, 1103 [1962].
}

systematische Untersuchungen über Olefine sind bisher noch nicht durchgeführt worden.

Im Rahmen der Serie feldionen-massenspektrometrischer Untersuchungen ${ }^{3-5}$ wird in der vorliegenden Arbeit über die FI-Massenspektren der 1Olefine vom Buten bis zum Okten sowie Decen, Dodecen und Tetradecen berichtet. Außerdem werden einige Isomere des Hexens und des Heptens auf ihr Verhalten im FI-Massenspektrometer eingehend untersucht.

${ }^{4}$ H. D. Beckey u. G. W [1965].

5 H. D. Beckey u. P. Schulze, Z. Naturforschg. 20 a, 1329 [1965]. 
Die verwendete Apparatur unterscheidet sich nicht von dem früher beschriebenen Gerät. Als Feldanoden wurden Platinspitzen von einigen $1000 \AA$ Krümmungsradius verwendet.

\section{Ergebnisse und Diskussionen}

\section{1-Olefine}

Die FI-Massenspektren einiger Olefine finden sich als Strichspektren in Abb. 1. Die Spektren des Äthylens und Propens sind in Abb. 1 nicht aufgeführt, weil sie unter den gleichen Versuchsbedingungen praktisch nur die Molekülionen aufweisen (bezüglich des Spektrums von 1-Buten s. Tab. 4). Die Bruchstückspektren der Olefine lassen sich unter Berücksichtigung einiger weniger Gesichtspunkte erklären:

1. Es findet selbst bei hohen Feldstärken keine Felddissoziation der $\mathrm{C}=\mathrm{C}$-Doppelbindung bei unverzweigten Olefinen statt.

Das bedeutet, daß man bei allen 1-Olefinen keine Massenlinie der Masse 14 beobachtet. Da dieser Befund bei allen Olefinen bestätigt wird, muß man die Massenlinie bei $m / e=14$, die beim Äthylen bei sehr hohen Feldstärken mit einer Intensität von $0,1 \%$ derjenigen des Molekülions auftritt, dem doppeltionisierten Molekülion zuschreiben. Das ist um so wahrscheinlicher, als man auch bei anderen Olefinen, allerdings auch nur in gleich geringem Maße, bei sehr hohen Feldstärken zweifachionisierte Molekülionen beobachten kann. Hier kann die Verwendung deuterierter Olefine eine endgültige Klärung herbeiführen.

Eine weitere Folgerung dieser Regel ist, daß man bei keinem 1-Olefin Ionen der Masse $M$-14 findet ( $M=$ Molekülion), sofern es keine Verunreinigung durch das nächst niedrigere Olefin enthält.

2. Es findet im allgemeinen keine Fragmentierung derjenigen $\mathrm{C}-\mathrm{C}$-Bindung statt, die $\operatorname{der} \mathrm{C}=\mathrm{C}$ Doppelbindung direkt benachbart ist.

In der Elektronenstoß-Massenspektrometrie ist diese Regel in abgeschwächter Form ebenfalls bekannt: „Ein Bruch derjenigen Bindungen, die direkt einer Doppelbindung benachbart sind (in $\alpha$-Stellung zu einer solchen Bindung), ist nicht so wahrschein-

B J.H. Beynon, Mass Spectrometry and its Applications to Organic Chemistry, Elsevier Publ. Co., Amsterdam 1960. lich wie der Bruch einer Einfachbindung in $\beta$-Stellung zu einer Doppelbindung “ 6 .

Eine Erklärung hierfür kann in den unterschiedlichen Bindungslängen und damit in dem partiellen Doppelbindungscharakter dieser Bindung gefunden werden. Für die C - C-Abstände und die Trennungsenergien der einzelnen Bindungen gelten die Werte der Tab. 1. Es ist anzunehmen, daß die Trennungsenergie für die der Doppelbindung benachbarte Bindung über $100 \mathrm{kcal} / \mathrm{Mol}$ beträgt.

\begin{tabular}{|r|c|c|}
\hline Bindung & $\AA$ & $\mathrm{kcal} /$ Mol \\
\hline $\mathrm{C}-\mathrm{C}$ & 1,54 & 83 \\
$\mathrm{C}-\mathrm{C}$ & 1,47 & 147 \\
$\mathrm{C}=\mathrm{C}$ & 1,33 & 147 \\
\hline
\end{tabular}

Tab. 1. C-C-Bindungs-Abstände und -Energien.

Diese Regel gilt sehr weitgehend. So findet man beim Propylen erst bei sehr hohen Feldstärken eine Massenlinie bei $m / e=27$ mit einer relativen Intensität von weniger als $0,05 \%$ und beim Buten mit einer relativen Intensität von weniger als $1 \%$. Auch bei allen übrigen Olefinen liegt die relative Intensität der Masse 27 weit unter $1 \%$, solange keine starke Adsorptions-Schichtbildung auf der Emissionsspitze vorliegt. Dann können allerdings Reaktionen auftreten, die zu Ionen der Massen 27 und M-27 führen, und die mit einer Intensität bis zu 5\% beobachtet werden können.

Mit Hilfe der beiden angeführten Regeln ist das Feldionen-Massenspektrum des Butens leicht zu erklären. Wie bei den n-Paraffinen wird ein MethylRadikal oder -Ion nur bei hohen Feldstärken abgespalten. Man findet daher erst bei hohen Feldstärken Ionen der Massen 15 und $M-15=41$. Die Intensität dieser Fragmentation steigt allerdings stärker als z. B. bei Paraffinen mit Erhöhung der Feldstärke an. Dieses Verhalten ist auf eine allgemein gültige Erscheinung zurückzuführen.

Die Stabilität sowohl des geladenen als auch des ungeladenen Fragments kann für das Auftreten gewisser Bruchstückionen maßgebend sein. Diese Regel gilt sowohl in der Elektronenstoß-Massenspektrometrie ${ }^{6}$ als auch in der Feldionen-Massenspektrometrie $^{7}$.

Im vorliegenden Fall handelt es sich um die besondere Stabilität des $\mathrm{C}_{3} \mathrm{H}_{5}$-Radikals bzw. seines

${ }^{7}$ H. D. BeCKey, Z. Naturforschg. 19 a, 71 [1964]. 

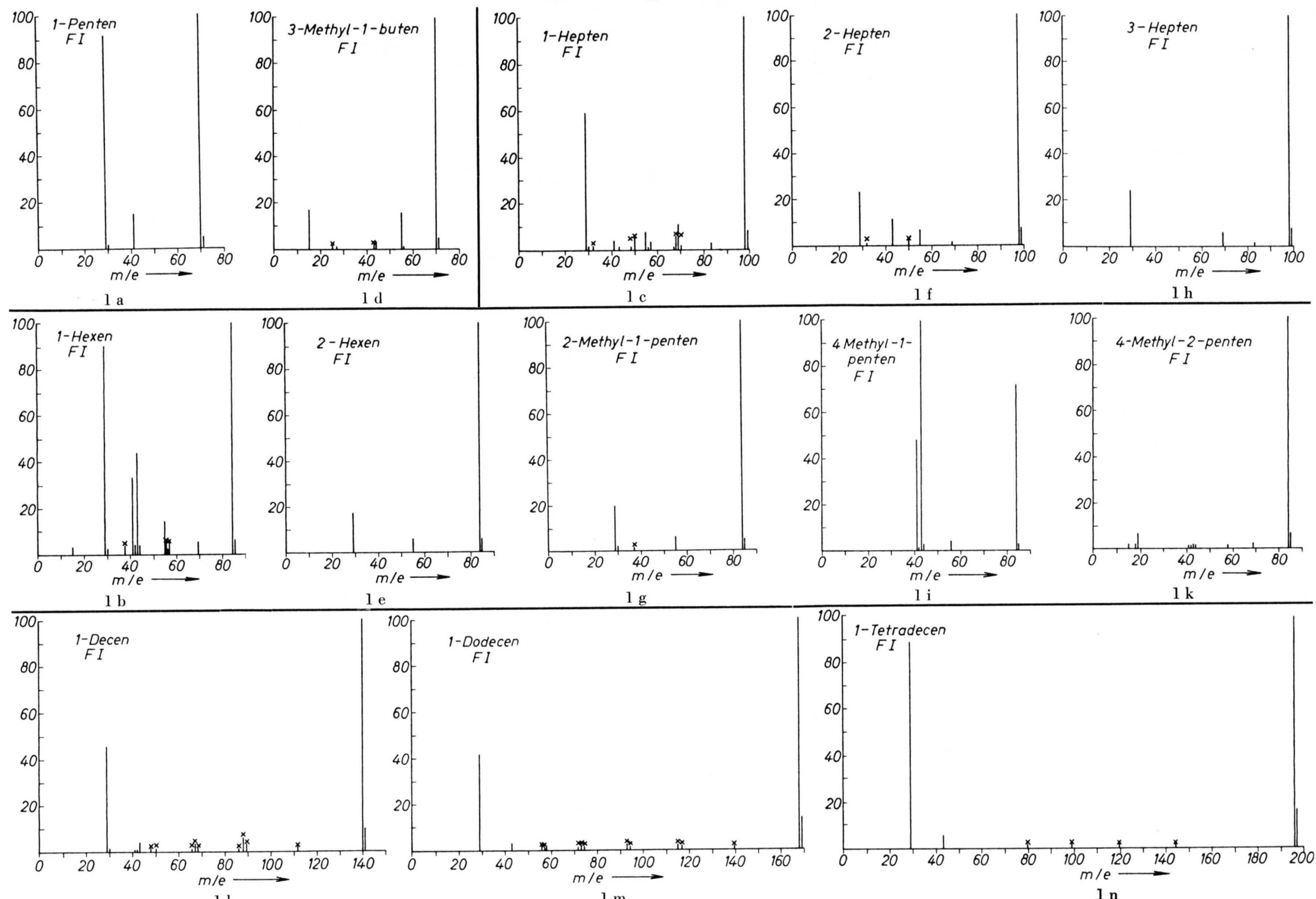

$1 \mathrm{~m}$

$1 \mathrm{n}$

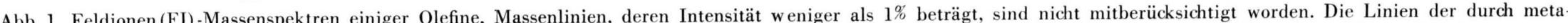
stabilen Zerfall entstehenden Ionen sind durch Kreuze gekennzeichnet. (Die Isotopenpeaks bei $m / e=30$ sind für 1-Dodecen- und 1-Tetradecen hinzuzufügen.) 
Ions. Swarc und Mitarbeiter ${ }^{8}$ haben gefunden, daß, das $\mathrm{C}_{3} \mathrm{H}_{5}$-Radikal, dem die beiden folgenden Grenzstrukturen zugeschrieben werden können,

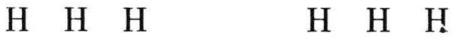

$$
\begin{aligned}
& \mathrm{C}=\mathrm{C}=\mathrm{C}^{\cdot} \longleftrightarrow{ }^{\circ} \mathrm{C}-\mathrm{C}=\mathrm{C} \\
& \begin{array}{llll}
\mathrm{H} & \mathrm{H} & \mathrm{H} & \mathrm{H}
\end{array}
\end{aligned}
$$

durch eine Resonanzenergie von $25 \mathrm{kcal} / \mathrm{Mol}$ besonders gut stabilisiert ist. Man kann annehmen, daß das $\mathrm{C}_{3} \mathrm{H}_{5}{ }^{+}$-Ion ähnlich gut stabilisiert ist.

Für die Olefine kann man die Regel daher in folgender Weise spezialisieren:

3. Die besondere Stabilität des $\mathrm{C}_{3} \mathrm{H}_{5}$-Radikals sowie des $\mathrm{C}_{3} \mathrm{H}_{5}{ }^{+}$-Ions muß bei allen Fragmentierungsprozessen für Olefine berücksichtigt werden.

Für energetische Betrachtungen muß man beachten, daß der Wert von $25 \mathrm{kcal} / \mathrm{Mol}=1,1 \mathrm{eV}$ für das freie Radikal gilt. Wenn $\psi_{1}$ und $\psi_{2}$ die beiden Eigenfunktionen bedeuten, welche die Grenzstrukturen des Allylions beschreiben, so wird im hohen elektrischen Feld der Beitrag einer der beiden Funktionen zur Gesamteigenfunktion

$$
\psi=c_{1} \psi_{1}+c_{2} \psi_{2}
$$

kleiner sein, so daß der Wert der Resonanzenergie geringer als im feldfreien Zustand wird.

Aus den Energieberechnungen, die Tab. 3 zugrunde liegen, folgt, daß bei Berücksichtigung der Spaltungsprozesse vieler verschiedener Olefinmoleküle ein mittlerer Wert von $0,7 \mathrm{eV}$ für die Resonanzenergie des Allylions in einem Felde von 0,5 bis $1,0 \cdot 10^{8} \mathrm{~V} / \mathrm{cm}$ am besten mit der Erfahrung übereinstimmt. Wegen der großen Stabilität des Allylions muß bei hohen Feldstärken das Methylion bzw. das Allylion im Spektrum des Butens stärker ansteigen, als man es für die Abspaltung eines Methylradikals oder Methylions bei anderen Olefinen oder bei n-Paraffinen erwartet. Viele andere Besonderheiten der Feldionen-Massenspektren der Olefine lassen sich unter besonderer Berücksichtigung der Stabilität des Allylradikals erklären.

a) Bei den Olefinen wird wie bei den Paraffinen beobachtet, daß diejenige Feldstärke $F_{(1)}$, die nötig ist, um die Intensität des $\mathrm{C}_{2} \mathrm{H}_{5}{ }^{+}$-Ions gleich derjenigen des Molekülions zu machen, mit zunehmender Kettenlänge des Olefins abnimmt. $F_{(1)}$ ist jedoch für

8 A. H. Sehon u. M. Swarc, Proc. Roy. Soc., Lond. A 202, [1950].
Penten niedriger als für Hexen und Hepten, obwohl es - wie bei den Paraffinen - höher liegen sollte. Das läßt sich dadurch erklären, daß durch die Abspaltung eines $\mathrm{C}_{2} \mathrm{H}_{5}{ }^{+}$-Ions beim Penten gerade das stabile Allylradikal entsteht, wodurch die Bildung eines $\mathrm{C}_{2} \mathrm{H}_{5}^{+}$-Ions begünstigt wird. Auf diese Weise ist ebenfalls der ungewöhnlich rasche Anstieg der Intensitäten der Massen 29 und 41 mit Erhöhung der Feldstärke zu erklären (Abb. 2).

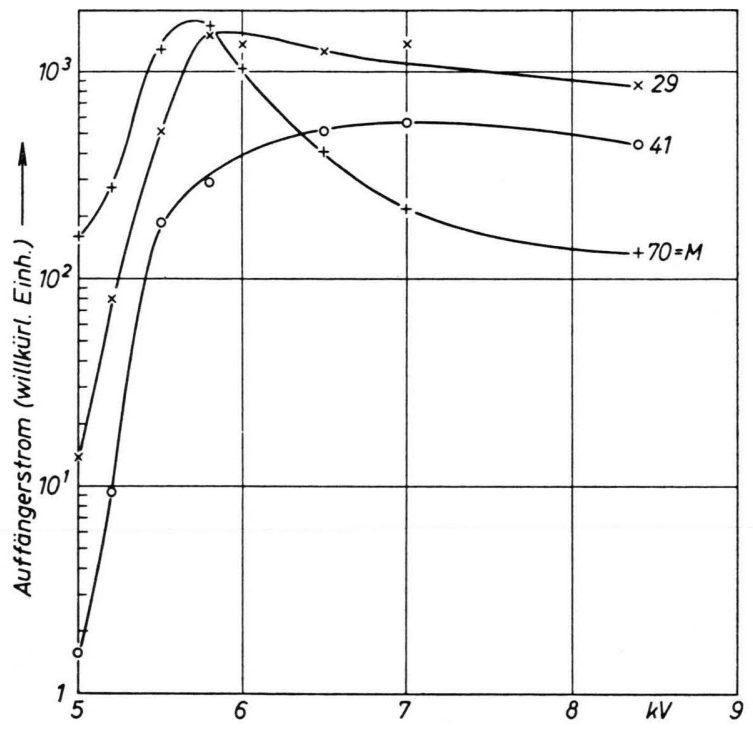

Abb. 2. Feldstärkeabhängigkeit der Absolutintensitäten der Ionenströme im FI-Massenspektrum des 1-Pentens.

Durch eine Energieabschätzung läßt sich der Ort maximaler Dichte positiver Ladung in Olefinmolekülen näherungsweise bestimmen.

Dafür ist die Kenntnis der Ionisierungsenergie der Olefine sowie die Ionisierungsenergie bestimmter Bindungen in den Olefinen erforderlich. Da die letzteren nicht bekannt und theoretisch nicht eindeutig definiert sind ${ }^{7}$, wird annähernd für die Ionisierungsenergie einer $\alpha$-Bindung die des Äthans, für eine $\beta$-Bindung die des Butans usw. eingesetzt. Dies ist nur ein sehr qualitatives Verfahren, dessen Berechtigung jedoch daraus abgeleitet werden kann, daß einerseits LoRquet und $\mathrm{HALL}^{9}$ quantenmechanisch mit Hilfe der erweiterten equivalent orbitalTheorie für n-Paraffine im hohen elektrischen Feld etwa die gleiche Ladungsverteilung wie nach dieser Abschätzungsmethode fanden und $\mathrm{da} ß$ andererseits

9 J. C. Lorquet u. G. G. Hall, Advances in Mass Spectrometry, Vol. III [1965], im Druck; Mol. Phys. 9, 29 [1965]. 
diese Ladungsverteilung zu Dissoziationsprozessen führt, die mit dem beobachteten übereinstimmen. Es sei für alle Olefine angenommen, daß die Ionisation ohne Feld an der Doppelbindung erfolgt und daß die Moleküle bevorzugt mit der Doppelbindung zur Spitze hin und wie die Paraffine weitgehend parallel zu den Feldlinien ausgerichtet sind.

Es werden die in Tab. 2 angegebenen Ionisierungsenergien für den Grundzustand benutzt. Die

\begin{tabular}{|ll|ll|}
\hline 1-Buten & $9,72 \mathrm{eV}$ & Äthan $11,65 \mathrm{eV}$ \\
1-Penten & $9,66 \mathrm{eV}$ & Butan $10,8 \mathrm{eV}$ \\
1-Hexen & $9,59 \mathrm{eV}$ & Hexan $10,43 \mathrm{eV}$ \\
2-Hexen & $9,16 \mathrm{eV}$ & Oktan $10,24 \mathrm{eV}$ \\
2-Methyl-1-penten & $9,60 \mathrm{eV}$ & & \\
1-Hepten & $9,54 \mathrm{eV}$ & & \\
2-Hepten & $9,13 \mathrm{eV}$ & & \\
3-Hepten & $9,10 \mathrm{eV}$ & & \\
1-Okten & $9,52 \mathrm{eV}$ & & \\
2-Okten & $9,11 \mathrm{eV}$ & & \\
\hline
\end{tabular}

Tab. 2. Ionisierungsenergien von Kohlenwasserstoffen.

Abstände der einzelnen Bindungen von $\operatorname{der} \mathrm{C}=\mathrm{C}$ Bindung können der Abb. 3 entnommen werden. Für den Energiegewinn bei der Verschiebung der

$$
\text { Spitze } C=C \frac{1}{\delta} c \frac{2.55 \AA}{\gamma} c \frac{+1.25 \AA-1}{\beta} c-c
$$

Abb. 3. Orientierung eines 1-Hexenmoleküls im hohen elektrischen Feld vor einer Spitze.

positiven Ladung im elektrischen Feld von der Doppelbindung um einen Betrag $\Delta x$ zum spitzenfernen Ende des Moleküls ergibt sich ${ }^{7}$

$$
\Delta E=\Delta I-E_{\mathrm{F}}=I_{2}-I_{1}-e F \Delta x .
$$

Diese Werte $\Delta E$ sind für zwei verschiedene Feldstärken in Tab. 3 angegeben. Falls für die Bildung eines Allylradikals eine Resonanzenergie $R$ einbezogen werden kann, ist das in Spalte $\Delta E-R$ berücksichtigt.

Für die Abspaltung eines Allylradikals aus 1Penten ist zusätzlich ein Gewinn an Resonanzenergie von $0,7 \mathrm{eV}$ in Rechnung zu stellen, der bei Abspal. tung eines Butylenradikals aus Hexen nicht auftritt. Die Bildung von $\mathrm{C}_{2} \mathrm{H}_{5}{ }^{+}$-Ionen aus 1-Penten ist daher im Vergleich zur Bildung aus 1-Hexen bei $F=0,5 \mathrm{~V} / \mathrm{cm}$ energetisch begünstigt, wie Tab. 3 zeigt.

Experimentell findet man entsprechend, daß $\mathrm{C}_{2} \mathrm{H}_{5}{ }^{+}$-Ionen mit größerer Häufigkeit aus 1-Penten als aus 1-Hexen abgespalten werden (s. Abb. 2 und

\begin{tabular}{|c|c|c|c|c|c|c|}
\hline \multirow[t]{2}{*}{ Substanz } & \multirow{2}{*}{$\begin{array}{l}\text { Frag- } \\
\text { ment } \\
\text { Ion }\end{array}$} & \multirow[t]{2}{*}{$\Delta I$} & \multicolumn{3}{|c|}{$F=0,5 \mathrm{~V} / \AA$} & \multirow{2}{*}{$\begin{array}{l}F= \\
1 \mathrm{~V} / \AA \\
\Delta E-R\end{array}$} \\
\hline & & & $E_{\mathrm{F}}$ & $\Delta E$ & $\Delta E-R$ & \\
\hline \multirow{8}{*}{$\begin{array}{l}\text { 1-Penten } \\
\text { 1-Hexen } \\
\text { 2-Hexen } \\
\text { 2-Methyl- } \\
\text { 1-penten } \\
\text { 1-Hepten }\end{array}$} & & & & 4 & & $-2,11$ \\
\hline & & & & & & $-2,5$ ? \\
\hline & & & $-1,28$ & -0 & & $-2,4$ \\
\hline & $\mathrm{CoH}$ & & $-1,28$ & +0 & & $-1,6$ \\
\hline & $\mathrm{C}_{2} \mathrm{H}_{5}$ & + & $-1,28$ & $-0,08$ & $-0,78$ & $-2,05$ \\
\hline & & + & -3 & -1 & & $-4,2$ \\
\hline & $\mathrm{C}_{2} \mathrm{H}_{5}$ & & -2 & -1 & - & $-3,79$ \\
\hline & & & -1 & -1 & - & $-2,91$ \\
\hline \multirow{3}{*}{ 2-Hepten } & $\mathrm{C}_{4}$ & $+c$ & $-1,28$ & -0 & - & $-2,5$ \\
\hline & & +2 & $-2,53$ & $-0,01$ & $-0,01$ & $-2,53$ \\
\hline & $\begin{array}{l}\mathrm{C}_{2} \mathrm{H} \\
\mathrm{C}_{3} \mathrm{H}\end{array}$ & $\begin{array}{l}+1 \\
+1\end{array}$ & $\begin{array}{l}-1,9 \\
-1,28\end{array}$ & $\begin{array}{l}-0 \\
+0\end{array}$ & $\begin{array}{l}23 \\
68\end{array}$ & $\begin{array}{l}-2,13 \\
-1,95\end{array}$ \\
\hline \multirow[t]{2}{*}{ 3-Hepten } & & & $-1,9$ & +0 & $+c$ & $-1,25$ \\
\hline & & & $-1,28$ & $+0,42$ & $-0,28$ & $-1,55$ \\
\hline
\end{tabular}
Abb. 4).
Tab. 3. Energieänderung (in eV) für die Verschiebung der positiven Ladung von der Doppelbindung zur $\alpha-, \beta-, \gamma-, \delta$-Bindung bei zwei Feldstärken (Orientierung und Bezeichnung der Bindungen entsprechend Abb. 3). Erläuterung der Größen $\triangle I, E_{\mathrm{F}}, \Delta E$ und $R$ im Text.

Ferner entnimmt man Tab. 3, daß die Bildung von Propylionen gegenüber derjenigen von Äthylionen aus 1-Hexen wegen der Bildung des stabilen Allylradikals energetisch bevorzugt ist. Dieses Ergebnis wird ebenfalls experimentell bestätigt, wie Abb. 4 zeigt.

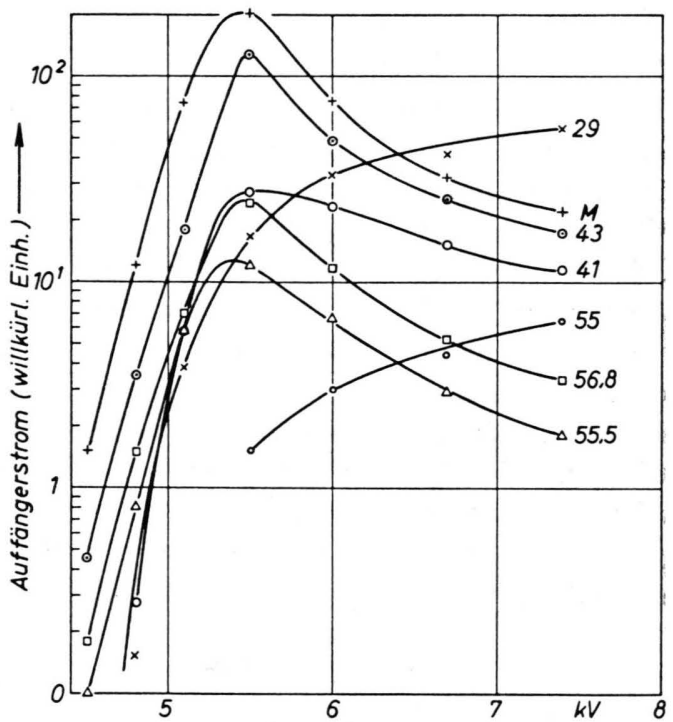

Abb. 4. Feldstärkeabhängigkeit der Absolutintensitäten der Ionenströme im FI-Massenspektrum des 1-Hexens.

b) Die Intensitäten der Fragmentionen beim 1Hepten in Abhängigkeit von der Feldstärke sind ebenfalls durch die Stabilität des Allylradikals bedingt. Die Massenlinie 29 ist im gesamten Feld- 
stärkebereich intensiver als die sämtlicher anderer Bruchstückionen. Das bedeutet, daß die Feldstärke groß genug ist, um die Ladung weitgehend an das Ende des Moleküls zu verschieben. Man findet außerdem, daß die Komplementärmasse $M-29=69$ relativ intensiv ist. Auffallend ist jedoch, daß nicht die Masse 43 und ihre Komplementärmasse M-43 $=55$ die nächst intensiven Massenlinien liefern, sondern das Allylion und das Koplementärion der Masse 57. Wäre das Auftreten bestimmter Ionen nur durch die Ladungsverschiebung ohne Berücksichtigung der Resonanzenergie des Allylions verursacht, so müßte man annehmen, daß $\mathrm{C}_{3} \mathrm{H}_{7}{ }^{+}$-Ionen mit größerer Wahrscheinlichkeit gebildet werden können als $\mathrm{C}_{4} \mathrm{H}_{9}{ }^{+}$. Ionen. Aus den Energiewerten der Tab. 3 kann man eine Ladungsdichteverteilung ableiten, die zu den experimentell gefundenen häufigsten Dissoziationsprozessen führt. Untersucht man die höheren 1-Olefine Okten, Decen, Dodecen und Tetradecen, so treten im Fragmentspektrum die für die niedrigen Olefine charakteristischen Massenlinien, wie etwa die der Massen 41 und 55, allmählich zurück und man kann ein den entsprechenden Paraffinen ähnliches Fragmentspektrum beobachten ${ }^{5}$. Dies ist darauf zurückzuführen, daß die Doppelbindung am Ende des Moleküls auf das Verhalten eines langen, gestreckten Moleküls im hohen elektrischen Feld keinen wesentlichen Einfluß mehr hat.

So findet man beim 1-Okten noch Ionen der Massen 41, 55 und 69 sowie der Massen 29, 43, 57 und 71 im Fragmentionenspektrum. Beim 1-Decen treten im wesentlichen nur noch die Ionen der Massen 29 und 43 auf, während alle übrigen Fragmente unter $1 \%$ der Intensität des Molekülions bleiben. Einzig die weiter unten zu beschreibenden metastabilen Ionen können noch einige Prozent erreichen. Auch darin ähneln die höheren l-Olefine den höheren nParaffinen, bei denen man ebenfalls nur die Fragmentionen der Massen 29 und 43 findet und im übrigen ein intensives Spektrum der metastabilen Ionen beobachten kann.

\section{Isomere Olefine \\ a) 3-Methyl-1-buten}

Beim 3-Methyl-1-Buten ist zu erwarten, daß bei niedrigen Feldstärken wie beim 1-Buten zunächst nur Molekülionen gebildet werden, daß aber bei höheren Feldstärken die Abspaltung eines $\mathrm{CH}_{3}$-Radikals oder eines $\mathrm{CH}_{3}{ }^{+}$-Ions durch die Methylsub- stitution erleichtert wird. Das beim 3-Methyl-1-Buten auftauchende Fragmention der Masse 55 weist ebenso wie das Allylion eine Resonanzstabilisierung auf. Man findet dementsprechend, daß beim 3-Methy]l-buten die Ionen der Massen 15 und $M-15$ bei gleicher Feldstärke stärker vorhanden sind als beim 1-Buten. Aus den Tab. 4 und 5 kann man ebenfalls

\begin{tabular}{|r|c|c|c|}
\hline \multicolumn{1}{|c|}{$m / e$} & $9 \mathrm{kV}$ & $11 \mathrm{kV}$ & $13 \mathrm{kV}$ \\
\hline 15 & 0,1 & 1 & 30,5 \\
27 & 0,02 & 0,06 & 1 \\
41 & 0,1 & 0,8 & 26,6 \\
$M=56$ & 100 & 100 & 100 \\
\hline
\end{tabular}

Tab. 4. Relativintensitäten des 1-Butens bei verschiedenen Spannungen Spitze-Kathode.

\begin{tabular}{|c|c|c|c|}
\hline \multicolumn{1}{|c|}{$m / e$} & $9 \mathrm{kV}$ & $11 \mathrm{kV}$ & $12 \mathrm{kV}$ \\
\hline 15 & 1 & 16,7 & 35,7 \\
27 & 0,3 & 1,1 & 6,0 \\
55 & 1 & 16 & 28,6 \\
$M=70$ & 100 & 100 & 100 \\
\hline
\end{tabular}

Tab. 5. Relativintensitäten des 3-Methyl-1-butens.

entnehmen, daß die Relativintensitäten des $\mathrm{C}_{2} \mathrm{H}_{3}{ }^{+}$Ions beim 3-Methyl-1-buten wesentlich stärker als beim 1-Buten sind. Durch die Methylsubstitution ist die Abspaltung eines $\mathrm{C}_{2} \mathrm{H}_{3}{ }^{+}$-Ions beim 3-Methyl1-buten bevouzugt. Ganz allgemein gilt - wie noch an Hand weiterer Beispiele gezeigt wird - , daß die der $\mathrm{C}=\mathrm{C}$-Bindung benachbarte Bindung durch eine Methylsubstitution am $\alpha$-ständigen Kohlenstoffatom eine erhöhte Fragmentierungswahrscheinlichkeit erhält.

b) 2,4,4-Trimethyl-1-penten und 2,4,4-Trimethyl2 -penten

Stärker ist dieser Einfluß der Substituenten bei dem von $\mathrm{W}_{\mathrm{AGNER}}{ }^{2}$ untersuchten 2,4,4-Trimethyl-1penten und 2,4,4-Trimethyl-2-penten. Bei beiden Substanzen ist das Fragmention der Masse 57 im Spektrum am intensivsten und das der Masse 55 wird dazu in fast gleichem Verhältnis gefunden, obgleich im Falle der zweiten Substanz bei dieser Fragmentation die der Doppelbindung benachbarte C - C-Bindung aufgebrochen wird:

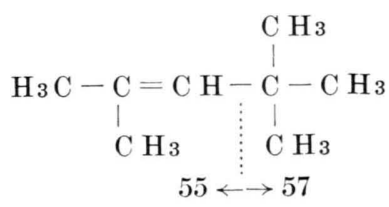


Allerdings wird durch die Verschiebung der Doppelbindung in $\beta$-Stellung das Molekül sehr viel stabiler gegenüber einem feldinduzierten Zerfall. Das beweist die sehr viel höhere Intensität, mit der das Molekülion beim 2,4,4-Trimethyl-2-penten beobachtet wird.

\section{c) Isomere des Hexens}

Weitere Beispiele für das Isomerieverhalten liefern die Isomeren des Hexens. Von diesen wurden folgende untersucht: 1-Hexen, 2-Methyl-1-penten, 4-Methyl-1-penten, 4-Methyl-2-penten und 2-Hexen.

Das 4-Methyl-1-penten

$$
\begin{aligned}
& \mathrm{H}_{2} \mathrm{C}=\mathrm{CH}-\mathrm{CH}_{2}-\underset{\mathrm{CH}}{\mathrm{C}-\mathrm{CH}_{3}} \\
& 41 \leftarrow \stackrel{?}{\longrightarrow} 43
\end{aligned}
$$

zerfällt besonders stark in die Fragmente der Massen 41 und 43 , weil die entsprechende C-C-Bindung sowohl durch die Stabilität des Allylfragments als auch durch die Verzweigung des $\beta$-ständigen Kohlenstoffatoms stark gelockert wird. Wie man der Tab. 6 entnehmen kann, sind schon bei einer we-

\begin{tabular}{|r|c|c|c|c|}
\hline$m / e$ & $\begin{array}{c}\text { 4-Methyl- } \\
2 \text {-penten } \\
9 \mathrm{kV}\end{array}$ & $\begin{array}{c}\text { 4-Methyl- } \\
1-\text {-penten } \\
7,5 \mathrm{kV}\end{array}$ & $\begin{array}{c}\text { 2-Methyl- } \\
\text { 1-penten } \\
9,3 \mathrm{kV}\end{array}$ & $\begin{array}{c}\text { 1-Hexen } \\
9 \mathrm{kV}\end{array}$ \\
\hline$M=84$ & 100 & 3,3 & 100 & 100 \\
$M-15=69$ & 2 & 2,4 & 0,05 & 4,4 \\
$M-29=55$ & - & - & 6,5 & 3 \\
$M-41=43$ & 1,7 & 100 & 0,5 & 28,3 \\
$M-43=41$ & 0,9 & 7,7 & 0,2 & 16,8 \\
$M-55=29$ & 0,7 & - & 20 & 13,8 \\
15 & 1,9 & 2,5 & 0,06 & 0,05 \\
\hline
\end{tabular}

Tab. 6. Relativintensitäten in den Feldionisations-Massenspektren einiger Isomere des Hexens.

sentlich geringeren Feldstärke als diejenige, die für die übrigen Substanzen angegeben ist, fast alle Molekülionen zerfallen. Die Hauptintensität liefern die $\mathrm{C}_{3} \mathrm{H}_{7}{ }^{+}$-Ionen.

Beim 2-Methyl-1-penten sind wegen der großen Felddissoziationswahrscheinlichkeit der $\beta$-Bindung und der Resonanzstabilisierung des $\mathrm{C}_{4} \mathrm{H}_{7}$-Radikals die $\mathrm{C}_{2} \mathrm{H}_{5}{ }^{+}$- und die $\mathrm{C}_{4} \mathrm{H}_{7}{ }^{+}$-Ionen weitaus am häufigsten. Man findet, daß die relative Intensität aller übrigen Fragmentionen unter 1\% bleibt.

Beim 4-Methyl-2-penten ist wegen der Resonanzstabilisierung des $\mathrm{C}_{5} \mathrm{H}_{9}^{+}$-Ions die Abspaltung von
Methylradikalen bzw. Methylionen ein wesentlich häufigerer Prozeß als bei den übrigen Isomeren.

Wie Tab. 3 ausweist, muß der Energiegewinn für die Verschiebung der positiven Ladung von der Doppelbindung zur $\beta$-Bindung (Abspaltung von $\mathrm{C}_{2} \mathrm{H}_{5}{ }^{+}$-Ionen) in der Reihenfolge 1-Hexen, 2-Methyl1-penten, 2-Hexen abnehmen. Nach Tab. 5 und 6 ändert sich die beobachtete relative Intensität der $\mathrm{C}_{2} \mathrm{H}_{5}^{+}$-Ionen in der gleichen Reihenfolge.

Obwohl die in Tab. 3 angegebenen Energiewerte eine qualitative Angabe des Ortes maximaler Dichte der positiven Ladung im Molekül erlauben, müssen nicht notwendigerweise die Fragmentierungswahrscheinlichkeiten eine Parallelität zu den maximalen Ladungsdichten aufweisen.

Trotzdem zeigt die Erfahrung daß, wenn bei zwei Fragmentierungsprozessen (1) und (2) der oben definierte Energiegewinn $\Delta E-R$ für den Prozeß (2) größer ist als für (1), auch gilt, daß die Fragmentierungswahrscheinlichkeit für (2) größer als für (1) ist.

Dies wurde soeben an einer größeren Zahl von Beispielen gezeigt, und man kann auch aus den Energiewerten der Tab. 3 noch weitere Einzelheiten herauslesen, die durch die in Tab. 7 wiedergegebenen experimentellen Werte der relativen Ionenintensitäten bestätigt werden. So ergibt sich z. B., daß die Intensität der $\mathrm{C}_{3} \mathrm{H}_{7}{ }^{+}$-Ionen aus 1-Hexen bei niedrigen und hohen Feldstärken größer als diejenigen der $\mathrm{C}_{2} \mathrm{H}_{5}{ }^{+}$-Ionen aus 2-Hexen sein sollte, und daß

\begin{tabular}{|c|c|c|c|c|c|c|}
\hline \multicolumn{4}{|c|}{1 -Hexen } & \multicolumn{3}{c|}{2 -Hexen } \\
$m / e$ & $7 \mathrm{kV}$ & $8 \mathrm{kV}$ & $9 \mathrm{kV}$ & $7 \mathrm{kV}$ & $8 \mathrm{kV}$ & $9 \mathrm{kV}$ \\
\hline 84 & 100 & 100 & 64 & 100 & 100 & 100 \\
69 & 3,4 & 6,8 & - & - & - & - \\
55 & 4,1 & 7,3 & 12,1 & - & 3,1 & 8,1 \\
43 & 37,5 & 63,6 & 35,9 & - & - & - \\
41 & 7,8 & 31,8 & 22,6 & - & - & - \\
29 & 6 & 79,1 & 100 & 3 & 9,9 & 27 \\
\hline
\end{tabular}

Tab. 7. Relativintensitäten in den FI-Massenspektren von 1-Hexen und 2-Hexen für verschiedene Feldstärken.

das Verhältnis $\mathrm{C}_{2} \mathrm{H}_{5}{ }^{+} / \mathrm{C}_{3} \mathrm{H}_{7}{ }^{+}$aus 1 -Hexen bei kleinen Feldstärken $<1$, bei hohen aber $>1$ sein sollte. Dies wird durch Tab. 7 bestätigt.

\section{d) Isomere des Heptens}

In Tab. 8 sind die relativen Intensitäten der Heptene für zwei verschiedene Feldstärken eingetragen. Bei niedrigen Feldstärken ist - wie Tab. 3 zeigt - beim 1-Hepten das $\mathrm{C}_{4} \mathrm{H}_{9}^{+}$-Ion wegen der Resonanzstabilisie- 


\begin{tabular}{|c|c|c|c|c|c|c|}
\hline & \multicolumn{2}{|c|}{ 1-Hepten } & \multicolumn{2}{c|}{ 2-Hepten } & \multicolumn{2}{c|}{ 3-Hepten } \\
$m / e$ & $7,6 \mathrm{kV}$ & $9 \mathrm{kV}$ & $7,6 \mathrm{kV}$ & $9 \mathrm{kV}$ & $7,6 \mathrm{kV}$ & $9 \mathrm{kV}$ \\
\hline 29 & 90 & 100 & 1,2 & 8,6 & 2,8 & 23,7 \\
41 & 4,8 & 6,6 & - & - & - & - \\
43 & 1,3 & 1,2 & 1,8 & 6,0 & - & - \\
55 & - & - & 0,9 & 3,2 & - & - \\
57 & 4,4 & 3,0 & - & - & - & - \\
69 & 4,4 & 3,2 & - & - & 0,2 & 5,3 \\
98 & 100 & 30,5 & 100 & 100 & 100 & 100 \\
\hline
\end{tabular}

Tab. 8. Relativintensitäten in den FI-Massenspektren von 1-Hepten, 2-Hepten und 3-Hepten für verschiedene Feldstärken.

rung des gleichzeitig gebildeten Allylradikals energetisch vor dem $\mathrm{C}_{3} \mathrm{H}_{7}^{+}$-Ion bevorzugt. Bei höheren Feldstärken ist das umgekehrte der Fall. Nach Tab. 8 weisen die relativen Ionenintensitäten beim 1-Hepten den gleichen Gang mit der Feldstärke auf.

Beim 2-Hepten wird wegen der Resonanzstabilisierung des 2-Butenylradikals bei niedrigen Feldstärken das $\mathrm{C}_{3} \mathrm{H}_{7}{ }^{+}$-Ion, bei höheren Feldstärken das $\mathrm{C}_{2} \mathrm{H}_{5}^{+}$-Ion häufiger gebildet.

Die Häufigkeit der Bildung von $\mathrm{CH}_{3}{ }^{+}$-Ionen sinkt in der Reihenfolge 1-Hepten, 2-Hepten, 3-Hepten. In der gleichen Reihenfolge sinken die Energiewerte der Tab. 3.

\section{Stabilität der Olefine gegenüber feldinduziertem Zerfall}

Es liegt nahe, einen Vergleich zwischen den Zerfallswahrscheinlichkeiten der Paraffine und der Olefine anzustellen. Von PAHL ${ }^{10}$ wurde die Zerfallswahrscheinlichkeit $W_{Z}$ eines Molekülions gegenüber Dissoziation in einer Elektronenstoß-Ionenquelle in der folgenden Weise definiert:

$$
W_{\mathrm{Z}}=I_{\mathrm{F}} /\left(I_{\mathrm{F}}+I_{\mathrm{P}}\right) .
$$

Darin bedeuten $I_{\mathrm{F}}$ die Intensitäten der Fragment. ionenströme und $I_{\mathrm{P}}$ die Intensität des Molekülionenstromes. Die Stabilität eines Molekülions ist dann gegeben durch

$$
W_{\mathrm{M}}=1-W_{\mathrm{Z}} \text {. }
$$

Úbernimmt man diese Definition für den Zerfall in einer Feldionenquelle, so kann man keinen festen Wert für $W_{\mathrm{M}}$ bei verschiedenen Feldstärken erwarten. Im Elektronenstoßexperiment ändert sich die Zerfallswahrscheinlichkeit oberhalb einer Elektronenenergie von etwa $30 \mathrm{eV}$ bis $70 \mathrm{eV}$ nur wenig ${ }^{10}$. Im Feldionen-Massenspektrometer nimmt die Häufigkeit der Molekülionen mit steigender Feldstärke ab und die Summe der Bruchstückionen zu. Das be-

10 M. PAHL, Z. Naturforschg. 9 b, 188, 418 [1954]. deutet, daß die Größe $W_{\text {M }}$ mit steigender Feldstärke sinkt.

Aus Abb. 5 und 6 erkennt man, daß in dem untersuchten Feldstärkebereich 2-Hexen stabiler ist als

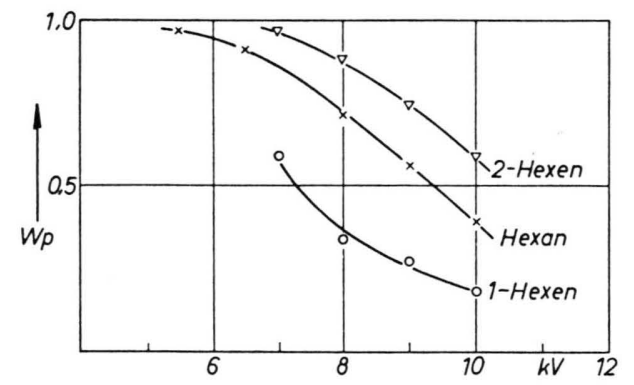

Abb. 5. Stabilität von Hexan, 1-Hexen und 2-Hexen gegenüber feldinduziertem Zerfall in Abhängigkeit von der Feldstärke.

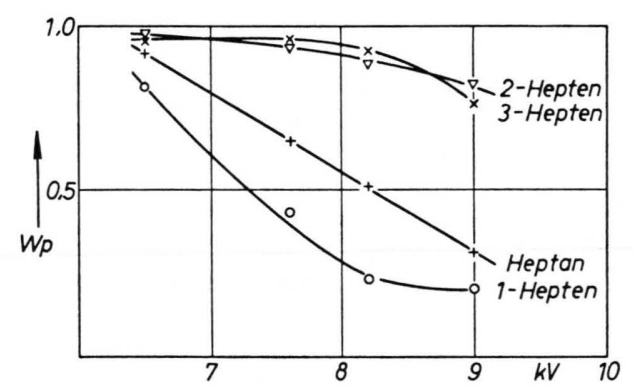

Abb. 6. Stabilität von Heptan sowie 1-, 2- und 3-Hepten gegenüber feldinduziertem Zerfall in Abhängigkeit von der Feldstärke.

Hexan, und dieses wieder stabiler als 1-Hexen. Auch bei den $\mathrm{C}_{7}$-Verbindungen ergibt sich die gleiche Reihenfolge wachsender Stabilität von 1-Hepten als dem instabilsten Molekülion über Heptan zu 2- und 3Hepten. Die beiden letzten Molekülionen unterscheiden sich kaum in ihrer Stabilität. Allerdings kann man feststellen, daß bei der Feldstärke, von der an die Stabilität des 3-Heptens stärker abnimmt als die des 2 -Heptens, eine intensive Bildung von $\mathrm{C}_{2} \mathrm{H}_{5}{ }^{+}$Ionen einsetzt.

Für 1-Penten und Pentan gilt auch, daß Pentan das stabilere Molekülion bildet. Bei l-Okten und Oktan läßt sich wegen Verunreinigungen in der 1-Oktenprobe keine eindeutige Aussage machen. Bei den höheren Olefinen wurde die Feldstärke nicht genügend stark variiert, um die entsprechenden Kurven angeben zu können. Es sei darauf hingewiesen, daß $\mathrm{P}_{\mathrm{AHL}}$ bis zum $\mathrm{C}_{8}$ eine zunehmende Stabilität in der Reihenfolge Alkane, Alkene gefunden hat, während sich ab $\mathrm{C}_{8}$ eine umgekehrte Reihenfolge ergibt. 
Für die Feldionen-Massenspektrometrie müßten systematische Untersuchungen hierzu bei reproduzierbaren Feldstärken vorgenommen werden.

\section{Metastabile Fragmentionen der Olefine}

Die metastabilen Zerfallsprozesse spielen bei den 1-Olefinen ebenfalls eine relativ große Rolle. Sie werden bei den Molekülen beobachtet, bei denen auch eine stärkere feldinduzierte Fragmentierung auftritt, d. h. vom 1-Penten ab. Bei den höheren Olefinen wird das Spektrum der metastabilen Ionen besonders deutlich sichtbar (s. Abb. 7). Dieses Verhalten entspricht ganz dem der n-Paraffine. Im übrigen treten aber insbesondere bei den niederen Olefinen, wie 1-Penten, 1-Hexen und 1-Hepten, charakteristische Unterschiede zu den n-Paraffinen auf. So findet man zunächst bis etwa zum 1-Hepten relativ intensiv metastabile Ionen, die durch Abspaltung eines neutralen Methylradikals entstehen (s. Tab. 9 und ${ }^{11}$ ).

Weiter beobachtet man außer der von den Paraffinen her bekannten Abspaltung von neutralen Alkylradikalen und Alkanen die Abspaltung von neutralen Olefinen. Bei den niederen Olefinen von 1-Penten bis 1-Okten wird vor allem Äthylen abgespalten. Beim 1-Hexen und 1-Hepten kann die Intensität der zugehörigen metastabilen Massenlinie bis zu 15\% der Molekülionenintensität erreichen. Beim Hepten wird ebenfalls eine starke Abspaltung eines Propenmoleküls beobachtet. Ab 1-Decen findet man die Ionen, die unter Abspaltung höherer Ole- fine entstehen. Die Intensitäten der metastabilen Ionen aus den isomeren Hexenen und Heptenen sind sehr viel kleiner als diejenigen aus 1-Hexen und 1-Hepten (siehe Tab. 9 und ${ }^{11}$ ).

\begin{tabular}{|c|c|c|c|c|c|c|}
\hline $\begin{array}{l}\text { abge- } \\
\text { spaltenes } \\
\text { Neutral } \\
\text { radikal }\end{array}$ & $\begin{array}{l}\stackrel{0}{0} \\
\stackrel{0}{\Xi} \\
0 \\
\stackrel{1}{1}\end{array}$ & 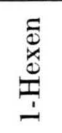 & 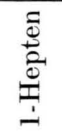 & 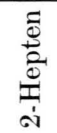 & 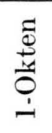 & 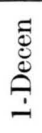 \\
\hline $\mathrm{CH}_{3}$ & 0,3 & 12,0 & 3,0 & 0,3 & 3,0 & 3,8 \\
\hline $\mathrm{C}_{2} \mathrm{H}_{3}$ & 0,02 & 1,5 & - & - & - & - \\
\hline $\mathrm{C}_{2} \mathrm{H}_{4}$ & 0,13 & 15,0 & 15,0 & 1,7 & 4,0 & 3,3 \\
\hline $\mathrm{C}_{2} \mathrm{H}_{5}$ & & & 5,0 & 0,4 & 8,0 & 6,2 \\
\hline $\mathrm{C}_{2} \mathrm{H}_{6}$ & & & 1,0 & 0,1 & 2,5 & 0,9 \\
\hline $\mathrm{C}_{3} \mathrm{H}_{5}$ & & & 1,7 & 0,2 & 1,1 & - \\
\hline $\mathrm{C}_{3} \mathrm{H}_{6}$ & & & 8,0 & 0,7 & 3,6 & 2,2 \\
\hline $\mathrm{C}_{3} \mathrm{H}_{7}$ & & & & & & 3,7 \\
\hline $\mathrm{C}_{3} \mathrm{H}_{8}$ & & & & & & 1,6 \\
\hline $\mathrm{C}_{4} \mathrm{H}_{7}$ & & & & & & 0,4 \\
\hline $\mathrm{C}_{4} \mathrm{H}_{8}$ & & & & & & 1,0 \\
\hline $\mathrm{C}_{4} \mathrm{H}_{9}$ & & & & & & 0,9 \\
\hline $\mathrm{C}_{4} \mathrm{H}_{10}$ & & & & & & 1,0 \\
\hline $\mathrm{C}_{5} \mathrm{H}_{9}$ & & & & & & 0,2 \\
\hline $\mathrm{C}_{5} \mathrm{H}_{10}$ & & & & & & 0,7 \\
\hline
\end{tabular}

Tab. 9. Relative Maximalintensität der Massenlinien metastabiler Fragmentionen aus Olefinen. Bei Berechnung der relativen Ionen-Häufigkeiten ist zu beachten, daß die Massenlinien metastabiler Ionen im Vergleich zu den Linien der übrigen Ionen etwa um einen Faktor 3 verbreitert sind.

Der Einfluß der Doppelbindung nimmt mit steigender Kettenlänge ab. Beim 1-Okten ist die Bildung von Äthylradikalen der intensivste metastabile Prozeß. Beim 1-Decen tritt die Abspaltung von $\mathrm{C}_{3} \mathrm{H}_{7^{-}}$und $\mathrm{C}_{4} \mathrm{H}_{9}$-Radikalen hinzu. Auch bei den Olefinen wird wie bei den Paraffinen beobachtet,

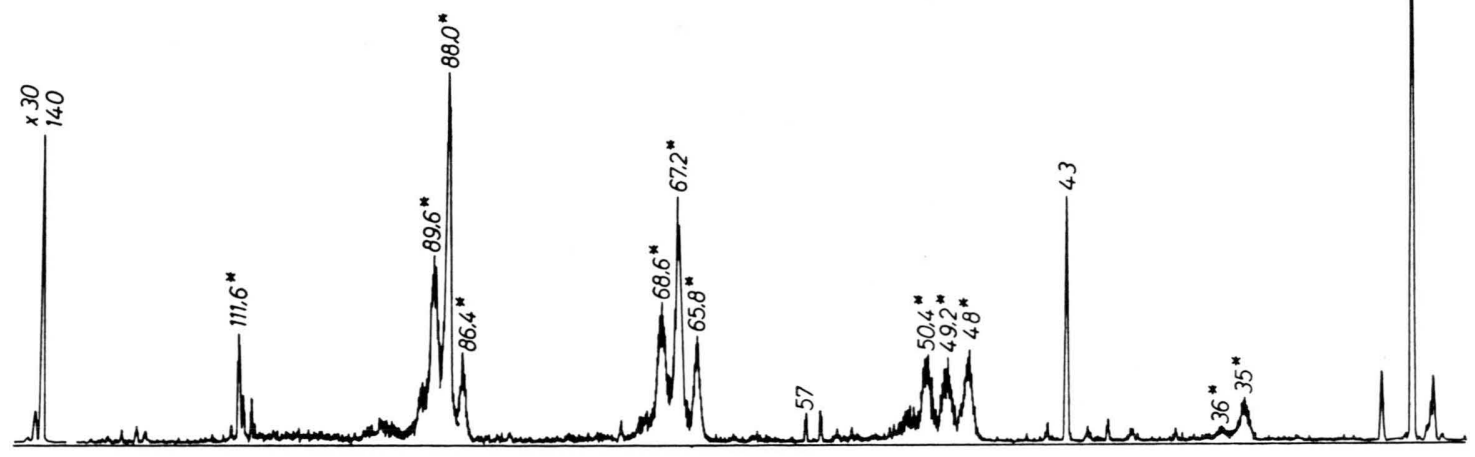

Abb. 7. Das FI-Massenspektrum des 1-Decens bei niedriger Feldstärke.

11 P. Schulze, Dissertation, Universität Bonn 1964. 
daß mit steigender Kettenlänge solche metastabilen Prozesse die Hauptintensität liefern, bei denen diese höheren Alkylradikale abgespalten werden.

Bei steigender Kettenlänge werden also die Spektren der Paraffine und Olefine hinsichtlich der Intensitätsverteilung der metastabilen Ionen ähnlicher, abgesehen davon, daß bei den Olefinen zusätzlich neutrale Alkene abgespalten werden.

In den FI-Massenspektren sehr langer Olefinmoleküle ist außer den Molekül-, Äthyl- und Propylionen nur das Spektrum metastabiler Ionen von nennenswerter Intensität (s. Abb. 7). Mit wachsen- der Feldstärke verschwindet dieses Metastabilenspektrum fast völlig zugunsten einer Vielzahl von Fragmentionen, die mit abnehmender Intensität von Masse 40 bis etwa Masse 86 reichen. In diesem Feldstärkebereich beträgt allerdings die Intensität des Molekülions nur noch wenige Prozent des $\mathrm{C}_{2} \mathrm{H}_{5}{ }^{+}$Ions und selbst die Intensität des $\mathrm{C}_{3} \mathrm{H}_{7}{ }^{+}$-Ions liegt unter $10 \%$ des Äthylions.

Der Deutschen Forschungsgemeinschaft sowie dem Verband der Chemischen Industrie danken wir für die großzügige Förderung der vorliegenden Arbeit durch finanzielle Unterstützung.

\title{
Die Bestimmung des effektiven lonisierungsquerschnitts in kondensierten Molekularstrahlen
}

\author{
Otto-Friedrich Hagena * und Wolfgang Henkes \\ Kernforschungszentrum Karlsruhe, \\ Institut für Kernverfahrenstechnik der Technischen Hochschule \\ (Z. Naturforschg. 20 a, 1344-1348 [1965] ; eingegangen am 26. Juli 1965)
}

\begin{abstract}
Clusters of condensed $\mathrm{CO}_{2}$-molecules that form a "condensed molecular beam" are being ionized by electron impact in the energy range $40 \mathrm{eV}<E_{\mathrm{el}}<580 \mathrm{eV}$. The average size of clusters is controlled by the pressure $p_{0}$ of $\mathrm{CO}_{2}$-gas in the nozzle of the beam source. By calibrating the apparatus with uncondensed $\mathrm{N}_{2}$-beams the ion currents can be converted into an effective cross section $\sigma_{\text {eff }}$ for ionization per molecule contained within the clusters relative to the corresponding cross section $\sigma_{0}$ of uncondensed $\mathrm{CO}_{2}$.

In the range covered by the experimental parameters it is found that always $\sigma_{\text {eff }} \leqq \sigma_{0}$. With increasing size of clusters $\sigma_{\text {eff }}$ tends to decrease and its maximum as function of electron energy moves to higher energies. At the highest value of $p_{0} \sigma_{\mathrm{eff}}=0.31 \sigma_{0}$ for $E_{\mathrm{el}}=150 \mathrm{eV}$, and the maximum of $\sigma_{\text {eff }}$ is situated at $290 \mathrm{eV}$ compared to $95 \mathrm{eV}$ for uncondensed $\mathrm{CO}_{2}$.
\end{abstract}

Durch Expansion eines Gases aus einer Düse ins Hochvakuum erhält man unter geeigneten Anfangsbedingungen kondensierte Molekularstrahlen ${ }^{1,2}$, bei denen die Atome oder Moleküle des Strahlgases zum größten Teil zu Agglomeraten zusammengelagert sind.

Vor einiger Zeit wurde vorgeschlagen, ionisierte und anschließend auf hohe Energie beschleunigte Agglomerate an Stelle von Molekülionen oder neutralen Atomen zum Aufbau eines Hochtemperaturplasmas $\mathrm{zu}$ verwenden ${ }^{3-5}$. In diesem Zusammenhang interessiert es, mit welchem Wirkungsgrad sich

* Z. Zt. Research Laboratories for The Engineering Sciences Thornton Hall, University of Virginia, Charlottesville, Va., USA.

1 E. W. Becker, K. Bier u. W. Henkes, Z. Phys. 146, 333 [1956].

2 E. W. Becker, R. Klingelhöfer u. P. Lohse, Z. Naturforschg. 17 a, 432 [1962].

3 W. Henkes, Z. Naturforschg. 17 a, 786 [1962]. kondensierte Molekularstrahlen ionisieren lassen, d. h. welcher Prozentsatz der in den kondensierten Agglomeraten vorhandenen Moleküle nach einer Ionisierung durch Elektronenstoß in ionisierten Agglomeraten enthalten ist.

Ein Wirkungsquerschnitt für Ionisation der Agglomerate kann nicht angegeben werden, da aus Untersuchungen der Massenverteilung von Agglomerat-Ionen bekannt ist, daß deren mittlere Masse bei steigender Elektronenenergie kleiner wird, daß also offensichtlich die Agglomerate bei der Ionisierung teilweise zerstört werden ${ }^{3,6-8}$. Man kann jedoch

4 W. Henkes, Phys. Letters 12, 322 [1964].

5 F. Prevot, private Mitteilung 1964.

6 R. E. Lechenby, E. J. Robbins u. P. A. Trevalion, Proc. Roy. Soc. London A 280, 409 [1964].

7 E. T. Greene u. T. A. Milne, J. Chem. Phys. 39, 3150 [1963].

8 J.Bauchert u. O. Hagena, Z. Naturforschg. 20 a, 1135 [1965]. 